\title{
Assessment of Minimal Residual Disease by Flow Cytometry in Acute Myeloid Leukaemia and its Correlation with Treatment Outcome
}

\section{Elsalakawy WA ${ }^{1 *}$, Abdelbary $\mathrm{HM}^{2}$, Youssef $\mathrm{SR}^{3}$ and Abdelmohsen $\mathrm{EA}^{4}$}

${ }^{1}$ Faculty of Internal Medicine, Department of Clinical Haematology and Bone Marrow Transplantation, Ain Shams University, Cairo, Egypt ${ }^{2}$ Faculty of Internal Medicine, Department of Clinical Haematology and Bone Marrow Transplantation, Ain Shams University, Cairo, Egypt ${ }^{3}$ Faculty of Clinical Pathology, Ain Shams University, Cairo, Egypt

${ }^{4}$ Faculty of Clinical Haematology and Bone Marrow Transplantation, Maadi Military Hospital, Cairo, Egypt

\begin{abstract}
Background: Acute myeloid leukaemia is the most common acute leukaemia in adults with increasing incidence, and despite major advances relapse is still a major concern. Assessing the response to therapy by determination of blast morphology is not sufficient to predict relapse in AML patients. Given its applicability to the majority of patients, FCM provides a powerful, independent prognostic factor to individualize therapy in AML patients.
\end{abstract}

Materials and methods: This study was conducted on 50 Egyptian adult AML patients. They were at day 28 post induction/post consolidation. FCM on bone marrow samples using FITC, PE, PC labelled CDs and a panel of antibodies tailored to each patient is used to assess MRD.

Results: Using a cut off value of $0.01 \%$ to assess MRD, $32 \%$ of patients had negative MRD and $68 \%$ had a positive MRD. In $58 \%$ of patients, lineage infidelity was used, CD19 was the most common marker used in $62 \%$ of patients while CD7 was used in $31 \%$ and CD56 was used in $7 \% .73 \%$ of cases with positive MRD had apparently normal initial cytogenetics while $62 \%$ of negative MRD cases had $t(8 ; 21)$. Collectively, $100 \%$ of MRD-ve cases are still in remission after 6 months of therapy while $61.8 \%$ of cases with positive MRD relapsed. MRD was in the lower range in cases with remission (0.545) and a higher range (0.44) in relapsed cases.

Conclusion: Our results indicate that measurement of MRD after induction/consolidation therapy provides a powerful prognostic factor for outcome in patients with AML.

Keywords: Minimal residual disease; Acute myeloid leukaemia; Flow cytometry; Bone marrow; Transplantation; Cytogenetics

\section{Introduction}

AML represents $80 \%$ of acute leukemias in adults and is the most common acute leukemia in this group of patients [1]. In 2015, over 20 thousand new cases were diagnosed and over 10000 patients succumb to the disease [2]. The incidence of AML increases with age, reaching 12.2 cases per 100 thousand population in people over 65 years. Advances in the understanding of the biology of the disease and treatment have improved the outcome for younger patients. Yet, the prognosis in the elderly who account for the main bulk of new cases remains poor [3]. Even with modern treatment options, nearly $70 \%$ of patients 65 years or older will die within 1 year of diagnosis [4]

Despite major advances in understanding the molecular pathogenesis of AML, evaluation of the bone marrow morphology remains the standard method to assess treatment response in clinical care and in the clinical trial setting, and is used to guide decisions of allogeneic transplantation. However, determination of blast percentage alone by light microscopy is hindered by limited sensitivity [5]

Although, conventional cytogenetics are undoubtedly important in the prognosis and risk stratification of AML, they require the presence of metaphase cells and hence, low sensitivity [6]. Interphase FISH improves sensitivity by approximately 10 fold compared with conventional cytogenetics.

It has been shown that FISH is more reliable than BM morphology for defining complete response. However, FISH cannot be performed in all patients and is only informative in cases with a known cytogenetic abnormalities that is detectable by a specific FISH probe [7].
Given these problems in using BM morphology to accurately assess remission status, there is accumulating evidence that detection of subclinical levels of leukemia (MRD) using MPFC or molecular-based strategies provides powerful independent prognostic information to help developing an individualized approach to treatment.

The aim of this study was to determine the value of MRD monitoring by FCM in adult acute myeloid leukemia patients post induction/ consolidation in order to determine efficacy of treatment, monitor remission status of the patient and predicting impending relapse.

\section{Patients and Methods}

This study was conducted on fifty Egyptian adult AML patients recruited from Ain-Shams University Hospitals, department of clinical hematology. They were at day 28 post induction/post consolidation. The study was carried on during the period from November 2015 till July 2016. Informed consent was obtained from all patients according to declaration of Helsinki.

*Corresponding author: Walaa Ali Elsalakawy, Associate Professor, Faculty of Internal Medicine, Department of Clinical Haematology and Bone Marrow Transplantation, Ain Shams University, Cairo, Egypt, Tel: +20 2 24821894; E-mail: drwalaa2010@gmail.com

Received April 09, 2018; Accepted August 08, 2018; Published August 10, 2018

Citation: Elsalakawy WA, Abdelbary HM, Youssef SR, Abdelmohsen EA (2018) Assessment of Minimal Residual Disease by Flow Cytometry in Acute Myeloid Leukaemia and its Correlation with Treatment Outcome. J Cancer Sci Ther 10: 205210. doi: 10.4172/1948-5956.1000545

Copyright: (c) 2018 Elsalakawy WA, et al. This is an open-access article distributed under the terms of the Creative Commons Attribution License, which permits unrestricted use, distribution, and reproduction in any medium, provided the original author and source are credited. 


\section{Inclusion criteria}

1) Patients included will be 18 years old or more.

2) No other malignancy.

3) No prior chemotherapy or radiotherapy. 4) No medical contraindications

\section{Exclusion criteria}

1) Patients with coexisting organ failure.

2) Bi-phenotypic acute leukemia

All subjects underwent the following:

\section{Laboratory investigations at initial diagnosis}

1. Bone marrow aspiration and examination of Leishman-stained BM smears

2. Flow cytometric Immunophenotyping (IPT): Flow cytometric IPT was performed on Coulter Navios-5-color flow cytometer (Coulter, Electronics, Hialeah, FL, USA), on BM samples using a standard acute leukemia panel of MoAb for all patients.

3. Initial cytogenetics (conventional cytogenetics in all patients and FISH for known AML cytogenetic abnormalities in selected patients).

\section{Laboratory investigations post-induction/post-consolidation}

1. Complete blood counts (CBC) using Sysmex (X5-500i) (Sysmex Cooperation, Cope, Japan), with examination of Leishmanstained PB smears.

2-BM aspiration on day 28 of induction or consolidation of treatment were obtained from all patients for

a. Examination of Leishman-stained BM smears: BM samples.

- Morphological complete remission was defined by the presence of less than $5 \%$ blast cells in the BM.

- Patients with partial remission were identified by the presence of $>5 \%$ blast cells, with typical morphology of myeloblasts.

b. FITC, PE, ECD, PC5 and PC7 labeled anti-CDs were used to evaluate MRD on blast cells gated on CD45 in all hematologically remitted patients. The choice of monoclonal antibodies used was tailored in each subject according to the original panel.

\section{Choice of the panel of CD markers for each patients}

- We created individualized MRD panel for each of our patients according to their initial CD markers (at diagnosis)

- The CD markers that we chose for each patient compromised common CDs for all patients like CD 45

- In addition to extra CDs tailored according to the original pattern at diagnosis to make sure we capture leukemia associated immunophenotype (LAIP) as possible

- we used leukemia-associated immunophenotype (LAIP) which included lineage-infidelity (cross-lineage lymphoid markers expression on myeloblasts) in the initial diagnostic samples of patients then we documented the persistence or disappearance of these LAIP after treatment.

- The second approach in LAIP is asynchronous antigen expression e.g. (co-expression of early markers like CD34 or CD117 with more mature markers).

\section{Sampling for IPT}

- Two mL PB samples were obtained on ethylenediamine tetraacetic acid, dipotassium salt ( $\mathrm{K}_{2}$-EDTA) in vacutainer tubes (final concentration of $1.5 \mathrm{mg} / \mathrm{mL}$ ) for $\mathrm{CBC}$

- $0.2 \mathrm{~mL}$ BM aspirate was obtained; smears were prepared and stained with Leishman's stain for morphological examination.

- One $\mathrm{mL} B M$ aspirate samples were obtained on $\mathrm{K}_{2}$-EDTA in vacutainer tubes for flow cytometric IPT.

\section{Flow cytometric IPT}

- FCM IPT of BM samples was done using Coulter Navios flow cytometer (Coulter, USA). A panel of MoAbs was used which included CD19, CD20, CD10, HLADR, CD38, CD34 and TdT. All MoAbs were supplied by Beckman Coulter (Fullerton, CA, USA). CD20, HLADR and TdT antibodies were fluorescein isothiocyanate (FITC) labeled while CD19, CD10, CD38 and CD34 were labeled by phycoerythrin (PE). Phycoerythrin-cyanine 5 (PC5) labeled CD45 was used for gating the hematogones. An additional panel was constructed for individual cases according to the initial panel at diagnosis. Special emphasis was made to include aberrant markers and leukemia associated phenotypes e.g. CD19, CD7, CD56 expression on myeloblasts.

- FITC and PE conjugated anti-Ig G1 were used as specific isotype matched controls for the used monoclonal antibodies.

- BM aspirate samples were processed on the same day of sample collection. They were counted using Sysmex Cell Counter and the total leucocyte count was adjusted to be around $5.0 \times 10^{9} / \mathrm{L}$ using phosphate buffered saline ( $8.5 \mathrm{~g} \mathrm{NaCL}, 1.07 \mathrm{~g} \mathrm{Na}_{2} \mathrm{HPO}_{4}$, $\left.0.39 \mathrm{~g} \mathrm{NaH}_{2} \mathrm{PO}_{4}-2 \mathrm{H}_{2} 0\right)$.

- $100 \mu \mathrm{L}$ of adjusted sample were aliquoted in the control tube as well as each sample tube and then $20 \mu \mathrm{L}$ of each MoAb or its isotype matched control were added.

- The control and test tubes were incubated for 15 minutes at room temperature, protected from light.

- After incubation, 1-2 mL of ammonium chloride-based erythrocyte lysing solution (82 $\mathrm{g}$ ammonium chloride, $10 \mathrm{~g}$ potassium bicarbonate, $0.37 \mathrm{~g}$ disodium EDTA) were added to every tube. Tubes were vortexed then analyzed using Coulter Epics XL flow cytometer (Coulter, Electronics, Hialeah, FL, USA).

\section{Interpretation of IPT patterns}

- CD45/side scatter gating strategy was adopted in the current study. Gating on blast cells was done in the region of dim CD45 and low side scatter.

- At least 100,000 or more events were gated for each sample.

- In initial flowcytometry results; Cells were considered positive for a marker when $>20 \%$ of the cells expressed that marker except for CD34 where expression by $10 \%$ of the cells was sufficient to confirm positivity.

- In MRD detection, limit of detection (LOD) was set according to values proposed in recent literature. Our instrument was set to 
detect even one in 1000.000 cells.

\section{Patient induction and consolidation chemotherapy}

$>$ ALL Patients received induction chemotherapy with $3+7$ chemotherapy using cytarabine $200 \mathrm{mg} / / \mathrm{m}^{2} / \mathrm{d}$ continuous intravenous infusion for 7 days with doxorubicin $25 \mathrm{mg} / \mathrm{m}^{2} / \mathrm{d}$ for 3 days followed by consolidation chemotherapy with highdose Ara-C 2-3 g/ $\mathrm{m}^{2}$ IV over 3 hours every 12 hours on days 1 , 3,5 for four cycles.

\section{Patients follow up (up to 6 months)}

$>$ All patients were followed up clinically and laboratory to determine whether they are in remission or relapsed. Bone marrow aspiration was done to all patients every 28 hours through follow up period.

\section{Statistical Analysis}

Data were collected, revised, coded and entered to the Statistical Package for Social Science (IBM SPSS) version 20. The quantitative data with parametric distribution were presented as mean, standard deviations and ranges while with non parametric distribution were presented as median with inter-quartile ranges (IQR) and also the qualitative data were presented as number and percentages. Chi-square test was used to compare between groups with quantitative data and Fisher exact test were used instead of chi square test only when the expected count in any cell found less than 5 . The comparison between two independent groups with quantitative data and parametric distribution was done by using Independent t-test. While comparison between two independent groups with quantitative data and nonparametric distribution was done by using Mann-Whitney test. Spearman correlation coefficients were used to assess the correlation between two quantitative parameters in the same group. Receiver operating characteristic curve (ROC) was used to assess the best cut off point with its sensitivity, specificity, positive predictive value (PPV), negative predictive value (NPV) and area under curve (AUC). The confidence interval was set to $95 \%$ and the margin of error accepted was set to $5 \%$. So, the p-value was considered significant as the following:

$\mathrm{p}>0.05$ : Non significant, $\mathrm{p}<0.05$ : Significant, $\mathrm{p}<0.01$ : Highly significant.

\section{Results}

This study was conducted on fifty Egyptian adult AML patients recruited from Ain-Shams University Hospitals, department of internal medicine, clinical hematology unit, Cairo, Egypt. They were at day 28 post induction/post consolidation. The most common AML type encountered in our study was AML M2 detected in $30 \%$ of patients followed by AML M0 detected in $28 \%$ of patients (Figure 1). While the most common cytogenetic abnormality was apparently normal cytogenetic in $58 \%$ of cases followed by $t(8 ; 21)$ in $28 \%$ of cases, (Figure 2) there is statistically significant correlation between the fate of cases (remission or relapse) and the diagnosis as $51.7 \%$ of cases in remission had AML (M2) and more than $66 \%$ of relapsed cases had AML (M0) (p-value <0.001). A significant correlation between the fate of cases and history (primary or on top of MDS) exists as $100 \%$ of cases in remission had 1ry disease and around $47 \%$ of relapsed cases was secondary to MDS (p-value 0.000) (Table 1). And there is highly statistically significant difference between the fate of cases and the initial cytogenetics as $100 \%$ of relapsed cases had normal cytogenetics

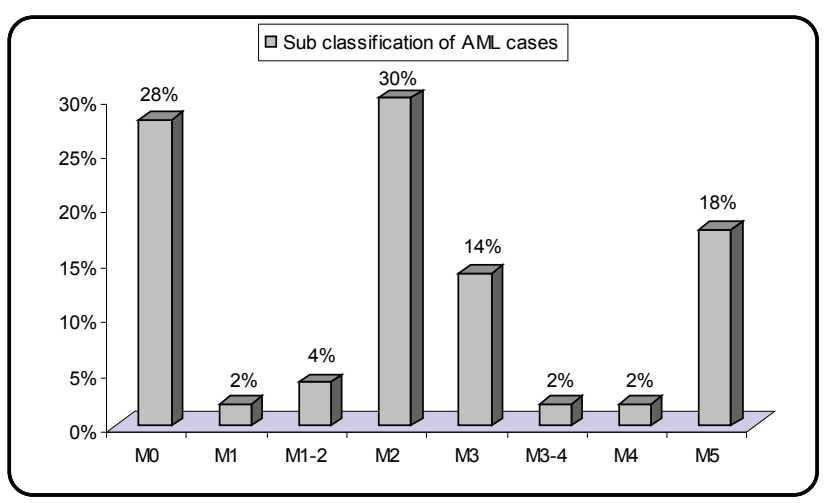

Figure 1: Distribution of the studied group as regard the diagnosis.

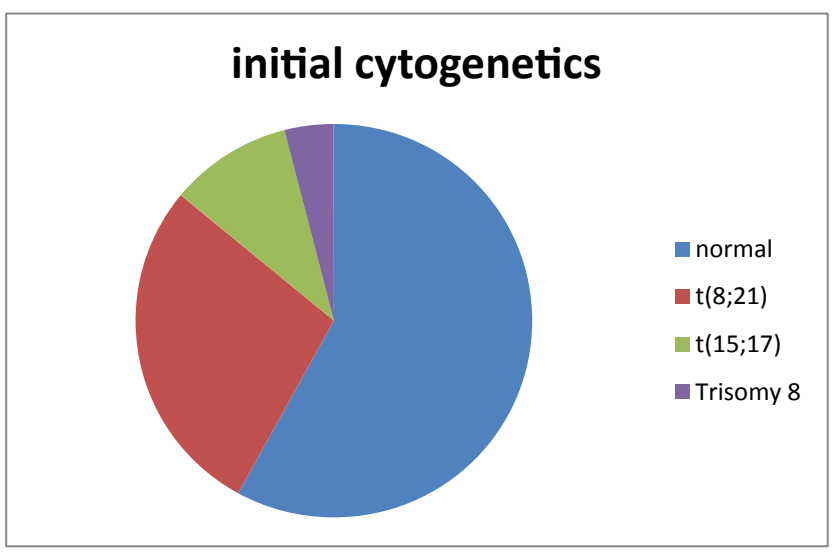

Figure 2: Distribution of the studied group as regard initial cytogenetics.

and $48.3 \%$ of cases in remission had positive translocation $t(8 ; 21)$ (p-value 0.000) (Table 2).

MRD was measured in $60 \%$ of cases post-induction and in $40 \%$ of cases post-consolidation. The number of cases with Negative MRD is $32 \%$ and $68 \%$ had Positive MRD with consideration that the cut off value of MRD is $0.01 \%$.

In $58 \%$ of patients Lineage infidelity was used, CD 19 was the most common marker used in $62 \%$ of this group, CD7 in $31 \%$ and CD56 was 7\% (Table 3).

In $100 \%$ of cases CD45 had been used, CD13 in 78\% of cases, CD19 in $36 \%$ and $18 \%$ used CD7 to detect MRD (Table 4 ).

There is statistically significant difference between MRD result and initial cytogenetic as $73.5 \%$ of cases with positive MRD had apparently normal initial cytogenetics and more than $62 \%$ of cases with negative MRD had Translocation $t(8 ; 21)$ ( $p$-value 0.001$) .100 \%$ of MRD negative cases are still in remission and $61.8 \%$ of cases with positive MRD relapsed with highly statistically significant p-value (0.001) (Table 5).

There is statistically significant correlation between fate of AML cases and MRD level post-induction as $100 \%$ of relapsed cases had a positive MRD ( $p$-value 0.004 ). There is statistically significant correlation between fate of cases and MRD level post-consolidation as $100 \%$ of relapsed cases had a positive MRD and $60 \%$ of cases in remission had negative MRD (p-value 0.011) (Table 6). 
Citation: Elsalakawy WA, Abdelbary HM, Youssef SR, Abdelmohsen EA (2018) Assessment of Minimal Residual Disease by Flow Cytometry in Acute Myeloid Leukaemia and its Correlation with Treatment Outcome. J Cancer Sci Ther 10: 205-210. doi: 10.4172/1948-5956.1000545

\begin{tabular}{|c|c|c|c|c|c|c|}
\hline \multirow{2}{*}{\multicolumn{2}{|c|}{ Sub classification of AML cases }} & \multicolumn{2}{|c|}{ Fate } & \multirow{2}{*}{ Total } & \multicolumn{2}{|c|}{ Chi-square } \\
\hline & & Remission & Relapsed & & $\mathrm{X}^{2}$ & P-value \\
\hline \multirow{2}{*}{ M0 } & $\mathrm{N}$ & 0 & 14 & 14 & \multirow{16}{*}{55.664} & \multirow{16}{*}{$<0.001^{*}$} \\
\hline & $\%$ & $0.00 \%$ & $66.70 \%$ & $28.00 \%$ & & \\
\hline \multirow{2}{*}{ M1 } & $\mathrm{N}$ & 0 & 1 & 1 & & \\
\hline & $\%$ & $0.00 \%$ & $4.80 \%$ & $2.00 \%$ & & \\
\hline \multirow{2}{*}{ M1-2 } & $\mathrm{N}$ & 0 & 2 & 2 & & \\
\hline & $\%$ & $0.00 \%$ & $9.50 \%$ & $4.00 \%$ & & \\
\hline \multirow{2}{*}{ M2 } & $\mathrm{N}$ & 15 & 0 & 15 & & \\
\hline & $\%$ & $51.72 \%$ & $0.00 \%$ & $30.00 \%$ & & \\
\hline \multirow{2}{*}{ M3 } & $\mathrm{N}$ & 7 & 0 & 7 & & \\
\hline & $\%$ & $24.14 \%$ & $0.00 \%$ & $14.00 \%$ & & \\
\hline \multirow{2}{*}{ M3-4 } & $\mathrm{N}$ & 1 & 0 & 1 & & \\
\hline & $\%$ & $3.45 \%$ & $0.00 \%$ & $2.00 \%$ & & \\
\hline \multirow{2}{*}{ M4 } & $N$ & 1 & 0 & 1 & & \\
\hline & $\%$ & $3.45 \%$ & $0.00 \%$ & $2.00 \%$ & & \\
\hline \multirow{2}{*}{ M5 } & $\mathrm{N}$ & 5 & 4 & 9 & & \\
\hline & $\%$ & $17.24 \%$ & $19.00 \%$ & $18.00 \%$ & & \\
\hline \multicolumn{7}{|c|}{ History } \\
\hline \multirow{2}{*}{ Primary } & $\mathbf{N}$ & 29 & 11 & 40 & \multirow{4}{*}{20.976} & \multirow{4}{*}{$0.000^{*}$} \\
\hline & $\%$ & $100.00 \%$ & $52.40 \%$ & $80.00 \%$ & & \\
\hline \multirow{2}{*}{ On top of MDS } & $\mathbf{N}$ & 0 & 10 & 10 & & \\
\hline & $\%$ & $0.00 \%$ & $47.60 \%$ & $20.00 \%$ & & \\
\hline
\end{tabular}

Table 1: Correlation between the fate of cases (remission, relapse) and the diagnosis, type of AML.

\begin{tabular}{|c|c|c|c|c|c|c|}
\hline \multirow{3}{*}{ Initial Cytogenetics } & \multicolumn{4}{|c|}{ Fate } & \multirow{3}{*}{$\begin{array}{c}\text { Chi square } \\
\qquad X^{2}\end{array}$} & \multirow{3}{*}{ P-value } \\
\hline & \multicolumn{2}{|c|}{ Remission } & \multicolumn{2}{|c|}{ Relapsed } & & \\
\hline & No. & $\%$ & No. & $\%$ & & \\
\hline Normal cytogentics & 8 & $27.6 \%$ & 21 & $100.0 \%$ & \multirow{4}{*}{27.463} & \multirow{4}{*}{$0.000^{* *}$} \\
\hline+ ve translocation $\mathrm{t}(8 ; 21)$ & 14 & $48.3 \%$ & 0 & $0.0 \%$ & & \\
\hline+ ve translocation $\mathrm{t}(15 ; 17)$ & 5 & $17.2 \%$ & 0 & $0.0 \%$ & & \\
\hline Trisomy 8 & 2 & $6.9 \%$ & 0 & $0.0 \%$ & & \\
\hline
\end{tabular}

Table 2: Correlation between the fate of cases (remission, relapse) and initial cytogenetics.

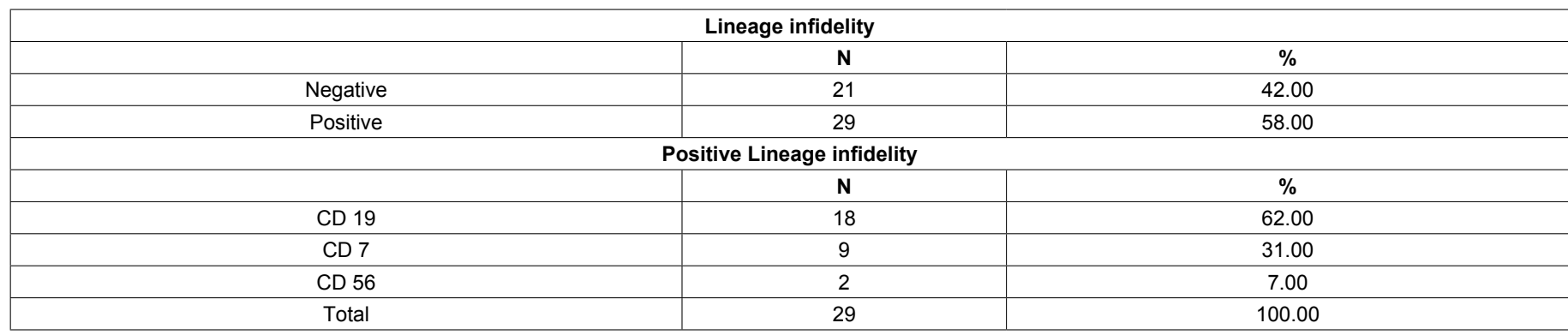

Table 3: Distribution of the studied group as regard lineage infidelity and most common markers used for lineage infidelity.

\begin{tabular}{|c|c|c|}
\hline Marker used & N & \% \\
\hline CD45 & 50 & 100.00 \\
\hline CD117 & 17 & 34.00 \\
\hline CD13 & 39 & 78.00 \\
\hline CD33 & 24 & 48.00 \\
\hline CD34 & 24 & 48.00 \\
\hline CD19 & 18 & 36.00 \\
\hline CD7 & 9 & 18.00 \\
\hline CD64 & 4 & 8.00 \\
\hline HLA-Dr & 2 & 4.00 \\
\hline MPO & 8 & 16.00 \\
\hline CD56 & 2 & 4.00 \\
\hline
\end{tabular}

Table 4: Distribution of the studied group as regard the markers used for MRD detection. 


\begin{tabular}{|c|c|c|c|c|c|c|c|c|c|}
\hline & \multirow{3}{*}{ Variables } & \multicolumn{6}{|c|}{ MRD } & \multirow{2}{*}{\multicolumn{2}{|c|}{ Chi-square }} \\
\hline & & \multicolumn{2}{|c|}{ Negative } & \multicolumn{2}{|c|}{ Positive } & \multicolumn{2}{|c|}{ Total } & & \\
\hline & & $\mathbf{N}$ & $\%$ & $\mathbf{N}$ & $\%$ & $\mathbf{N}$ & $\%$ & $x^{2}$ & P-value \\
\hline \multirow{4}{*}{ Initial cyto } & Negative & 4 & $25.00 \%$ & 25 & $73.50 \%$ & 29 & $58.00 \%$ & \multirow{4}{*}{15.936} & \multirow{4}{*}{0.001} \\
\hline & Translocation t(8;21) & 10 & $62.50 \%$ & 4 & $11.80 \%$ & 14 & $28.00 \%$ & & \\
\hline & Translocation $\mathrm{t}(15 ; 17)$ & 2 & $12.50 \%$ & 3 & $8.80 \%$ & 5 & $10.00 \%$ & & \\
\hline & Trisomy 8 & 0 & $0.00 \%$ & 2 & $5.90 \%$ & 2 & $4.00 \%$ & & \\
\hline \multirow{2}{*}{ Fate } & Remission & 16 & $100.00 \%$ & 13 & $38.20 \%$ & 29 & $58.00 \%$ & \multirow{2}{*}{14.597} & \multirow{2}{*}{$0.001^{*}$} \\
\hline & Relapsed & 0 & $0.00 \%$ & 21 & $61.80 \%$ & 21 & $42.00 \%$ & & \\
\hline
\end{tabular}

Table 5: Correlation between MRD results and initial cytogenetics, fate of cases (remission and relapsed).

\begin{tabular}{|c|c|c|c|c|c|c|c|}
\hline \multirow{3}{*}{ Variables } & & \multicolumn{4}{|c|}{ Fate } & \multirow{3}{*}{ Chi square } & \multirow{3}{*}{ P-value } \\
\hline & & \multicolumn{2}{|c|}{ Remission } & \multicolumn{2}{|c|}{ Relapsed } & & \\
\hline & & No. & $\%$ & No. & $\%$ & & \\
\hline \multirow{2}{*}{ MRD result post-induction } & Negative & 10 & $52.60 \%$ & 0 & $0.00 \%$ & \multirow{2}{*}{8.684} & \multirow{2}{*}{$0.004^{* *}$} \\
\hline & Positive & 9 & $47.40 \%$ & 11 & $100.00 \%$ & & \\
\hline \multirow{2}{*}{ MRD result post-consolidation } & Negative & 6 & $60.00 \%$ & 0 & $0.00 \%$ & 8.571 & \multirow{2}{*}{$0.011^{*}$} \\
\hline & Positive & 4 & $40.00 \%$ & 10 & $100.00 \%$ & FE\# & \\
\hline
\end{tabular}

Table 6: Correlation between fate of cases (remission and relapse) and MRD result post induction and post consolidation.

\begin{tabular}{|c|c|c|c|c|c|c|c|c|}
\hline \multirow{3}{*}{ Variables } & \multicolumn{6}{|c|}{ Fate } & \multirow{2}{*}{\multicolumn{2}{|c|}{ T-test }} \\
\hline & \multicolumn{3}{|c|}{ Remission } & \multicolumn{3}{|c|}{ Relapsed } & & \\
\hline & Mean & \pm & & Mean & \pm & SD & $\mathbf{t}$ & P-value \\
\hline MRD & 0.545 & \pm & 0.277 & 0.446 & \pm & 0.581 & -2.287 & $0.027^{*}$ \\
\hline
\end{tabular}

Table 7: Relation between MRD level and fate of cases (remission and relapsed).

There is statistically significant correlation between fate of AML cases and MRD as MRD had lower range in cases with remission (0.545) and higher range (0.446) with cases in relapse by using unpaired t-test (p-value 0.027) (Table 7).

\section{Discussion}

Previous research groups had found aberrant expression of cell surface antigens on leukemic blasts; these antigenic alterations are referred to as leukemia-associated immunophenotype (LAIP) and are the basis of immunologic MRD detection.

A study done by Al-Mawali et al. found that asynchronous expression of antigens usually seen at distinct stages of myeloid maturation (co-expression of early antigens such as CD34 and CD117 with more mature antigens such as CD15 or CD 65), as well as crosslineage antigen expression of lymphoid markers on myeloblasts (lineage-infidelity) represent the most common LAIPs encountered using five-color flowcytometry [8]. Identification of these LAIPs in the initial diagnostic sample of the patient then using MFC to document the persistence or disappearance of the LAIPs is a possible approach to monitor the minimal residual disease [9].

In our study, Lineage infidelity was used in 58\% of patients, CD19 was the most common marker used in $62 \%$ of this group, CD7 in $31 \%$ and CD56 in 7\%. This is in accordance with a study done by Liu et al. that show that the cross-lineage antigen and asynchronous antigen expression were the most frequent aberrant phenotypes. CD7, CD19 and CD56 expressing on CD34+ cells were major cross-lineage antigen [10]. Furthermore, In a pilot study by Mehta et al. to standardize MRD estimation in AML by flow cytometry at a cancer center in India on a 46 patients of AML, The commonest LAIP illustrated was the cross lineage infidelity in $78 \%$ of their patients, In their cohort they showed that the cross-lineage expression of CD56 was $43 \%$ and CD 4 was $36 \%$. In their study they used seven combinations, each containing five antibodies, with CD34 and CD45 as backbone [11]. In accordance, in our study CD45 markers was considered the backbone marker from start onwards, and this was the base of other previous experience as in Van Dongen [12] and Gaipa [13]. In addition CD19, CD20, CD10, HLADR, CD38, CD34 and TdT Were (FITC) labeled while CD19, CD10, CD38 and CD34 were labeled by (PE). (PC5) labeled CD45 was used for gating the hematogones.

One of the drawbacks of the approach of LAIP is that the immunophenotypic aberrancies of leukemic myeloblasts are not always stable at relapse, a phenomenon referred to as 'immunophenotypic shift'. In a study by Langerbrake et al. they found that the immunophenotype by flow cytomety differed at relapse by at least one antigen than that at presentation and that most cases showed an immature phenotype at relapse and that $72 \%$ of cases showed a gain of markers associated with lineage immaturity. This antigenic shift was observed in $88 \%$ of cases in their study [14]. So, the panel of antibody used for MRD monitoring should not be restricted to immunophenotype at presentation.

Second, access to initial samples may be unavailable and the construction of MRD panel that is individual for each patient may be difficult for some laboratories. So, a difference of normal approach which relies on using a healthy control bone marrow samples and studying characteristic sequential pattern of antigen expression for each lineage is delineated and the aberrant myeloblasts is detected by deviation from this normal pattern [15].

The ideal threshold level for positivity of MRD and timing of measurement in AML has not been established, and given the complexity of the disease, a single cut-off and timing for MRD could not be universally applied to all patients.

In our study, using a threshold of 0.01 , the number of cases with negative MRD is $32 \%$ and $68 \%$ had positive MRD. In $60 \%$ of patients, MRD was used post-induction while in the remaining $40 \%$ it is used post-consolidation. 
Citation: Elsalakawy WA, Abdelbary HM, Youssef SR, Abdelmohsen EA (2018) Assessment of Minimal Residual Disease by Flow Cytometry in Acute Myeloid Leukaemia and its Correlation with Treatment Outcome. J Cancer Sci Ther 10: 205-210. doi: 10.4172/1948-5956.1000545

Our study showed that MRD positive case was highly susceptible to relapse than MRD negative cases as $100 \%$ of MRD negative cases after follow up for 6 months are still in remission and $61.8 \%$ of cases with positive MRD relapsed ( $\mathrm{p}=0.001$ ). When divided, MRD measured post-induction showed that $52.6 \%$ of cases in remission were MRDve and $100 \%$ of relapsed cases were MRD+ve, while MRD measured post-consolidation showed that $60 \%$ of cases in remission were MRDve and again $100 \%$ of relapsed cases were MRD+ve. This comes in concomitance with data shown by Köhnke et al. who found that The 5 -year RFS was $16 \%$ for MRD-positive patients and $43 \%$ for patients with no evidence of residual disease $(p<0.001)$. And this is in addition to other known risk factors as initial cytogenetic as $48.3 \%$ of cases in remission had Translocation $\mathrm{t}(8 ; 21)$ and $100 \%$ of relapsed cases had normal karyotyping (intermediate risk) [16]. In accordance, $62.5 \%$ of MRD-ve cases in our study had $t(8 ; 21)$ and $73.5 \%$ of MRD +ve cases had apparently normal cytogenetics.

In our study, the mean MRD level was lower in patients in remission (0.545) compared to relapse patient (0.446) with statistically significant difference ( $\mathrm{p}$-value 0.027 ). However, lack of MRD may not predict maintenance of remission and while the presence of MRD may identify patients at increased risk of relapse, its absence has not been correlated with maintenance of remission.

\section{Conclusion and Future Perspectives}

As mentioned above, there have been contradictory findings regarding the most prognostically significant time point for measurement of MRD (that is, end of induction, end of consolidation, before or post SCT). However, what has consistently been shown is that when MRD is measured at almost any time point, its presence is correlated with an increased risk of relapse and worsened survival. In essence, MRD detection techniques must be sensitive enough to detect a low level of residual leukemic blasts. Al-Mawali et al. found that in a 54 AML patients using a five-color flow cytometry a minimum sensitivity of $10^{-4}$ was reached independent of the aberrant event tested and that the technique was highly reproducible [9].

A future perspective is MRD measurement at the time of allogeneic HSCT for AML patients is a powerful, independent predictor of adverse outcome post-transplant and that MRD monitoring posttransplant gives prognostically relevant information. However, it is unclear how MRD status measured at the time of transplant and later on after transplant could determine therapeutic decisions either by giving additional therapy to decrease tumor load before transplant or by using minimized immune-suppression to optimize GVL or withdrawal of immune-suppression to prevent relapse in patients with persistent MRD positivity post-transplant although currently data from controlled prospective trials are still missing. Thus, MRD detection provides a powerful, independent prognostic predictor of adverse outcomes both in AML patients receiving chemotherapy or in the transplant setting however, further studies are needed in the transplant patients.

\section{References}

1. Yamamoto JF, Goodman MT (2008) Patterns of leukemia incidence in the United States by subtype and demographic characteristics, 1997-2002. Cancer Causes Control 19: 379-390.

2. Siegel RL, Miller KD, Jemal A (2015) Cancer statistics, 2015. CA Cancer J Clin 65: 5-29.

3. Shah A, Andersson TM, Rachet B, Bjorkholm M, Lambert PC (2013) Survival and cure of acute myeloid leukaemia in England, 1971-2006: A populationbased study. Br J Haematol 162: 509-516.

4. Meyers J, Yu Y, Kaye JA, Davis KL (2013) Medicare fee-for-service enrollees with primary acute myeloid leukemia: an analysis of treatment patterns, survival, and healthcare resource utilization and costs. Appl Health Econ Health Policy 11: $275-286$.

5. Grimwade D, Freeman SD (2014) Defining minimal residual disease in acute myeloid leukemia: which platforms are ready for "prime time"? Blood 124: 3345-3355.

6. Fang M, Storer B, Wood B, Gyurkocza B, Sandmaier BM, et al. (2012) Prognostic impact of discordant results from cytogenetics and flow cytometry in patients with acute myeloid leukemia undergoing hematopoietic cell transplantation. Cancer 118: 2411-2419.

7. Bacher U, Kern W, Schoch C, Schnittger S, Hiddemann W, et al. (2006) Evaluation of complete disease remission in acute myeloid leukemia: a prospective study based on cytomorphology, interphase fluorescence in situ hybridization, and immunophenotyping during follow-up in patients with acute myeloid leukemia. Cancer 106: 839-847.

8. Al-Mawali A, Gillis D, Hissaria P, Lewis I (2008) Incidence, sensitivity, and specificity of leukemia-associated phenotypes in acute myeloid leukemia using specific five-color multiparameter flow cytometry. Am J Clin Pathol 129: 934-945.

9. Al-Mawali A, Gillis D, Lewis I (2009) The role of multiparameter flow cytometry for detection of minimal residual disease in acute myeloid leukemia. Am J Clin Pathol 131: 16-26.

10. Liu YR, Wang YZ, Chen SS, Chang Y, Fu JY, et al. (2007) Analysis of immunophenotype and leukemia associated immunophenotype in 610 patients with acute myeloid leukemia. Zhonghua Xue Ye Xue Za Zhi 28: 731-736.

11. Mehta P, Chopra A, Soni S, Kumar R (2015) Haematological malignancies 307P Standardizing MRD detection by flow cytometry in AML in a developing country. Annal Oncol 26: IX85-IX92.

12. Van Dongen JJ, Lhermitte L, Böttcher S, Almeida J, Van der Velden VH, et al. (2012) Euroflow antibody panels for standardized n-dimensional flow cytometric immunophenotyping of normal, reactive and malignant leukocytes. Leukemia 26: 1908-1975.

13. Gaipa G, Cazzaniga G, Valsecchi MG, Panzer-Grümayer R, Buldini B, et al. (2012 Time point-dependent concordance of flow cytometry and real-time quantitative polymerase chain reaction for for minimal residual disease detection in childhood acute lymphoblastic leukemia. Haematologica 97: 1582-1593.

14. Langebrake C, Brinkmann I, Teigler-Schlegel A, Creutzig U, Griesinger F, et al. (2005) Immunophenotypic differences between diagnosis and relapse in childhood AML: Implications for MRD monitoring. Cytometry B Clin Cytom 63: 1-9.

15. Jaso JM, Wang SA, Jorgensen PL, Lin P (2014) Multi-color flow cytometric immunophenotyping for detection of minimal residual disease in AML: Past present and future. Bone Marrow Transplant 49: 1129-1138.

16. Köhnke T, Sauter D, Ringel K, Hoster E, Laubender RP, et al. (2015) Early assessment of minimal residual disease in AML by flow cytometry during aplasia identifies patients at increased risk of relapse. Leukemia 29: 377-386. 